\title{
IDIOPATHIC HYPERTROPHIC OSTEOARTHROPATHY: CASE REPORT
}

\author{
Bruna Luiza Oliveira Limaa ${ }^{1, *}$, Mirian Parolo Ribeiro ${ }^{1}$, Fernanda da Costa Ferreira Guerra ${ }^{1}$, Fabiana de Castro Machado ${ }^{1}$, Camila \\ de Oliveira Trevisan Coutinho ${ }^{1}$
}

1. Universidade Federal de Mato Grosso, Sinop (MT), Brazil.

*Corresponding author: bbrunalima99@gmail.com

\section{BACKGROUND}

Hypertrophic osteoarthropathy $(\mathrm{HOA})$ is characterized by abnormal proliferation of the skin in the distal region of the extremities and periosteal proliferation of long bones. Secondary HOA is more frequent and usually associated with bronchogenic carcinoma, while primary HOA may be of genetic cause with prevalence in males or idiopathic, its rarest form. Among the clinical features is digital clubbing, periostosis of tubular bones, synovial effusion and cutis verticis gyrata. Bone scan, magnetic resonance and positron emission tomography can help in diagnosis. Therapy involves the use of nonsteroidal anti-inflammatory drugs, colchicine and corticotherapy for cases of primary genetic $\mathrm{HOA}$, treatment of the underlying disease for cases of secondary $\mathrm{HOA}$, and for the idiopathic form, there is still no consensus.

\section{CASE REPORT}

Female patient, 66 years old, sought rheumatologist for diffuse arthralgia, with inflammatory rhythm, associated with edema and heat in knees for 1 year, with improvement of the condition after use of injectable corticosteroids. Personal history of esophageal achalasia with surgery 20 years ago and hypothyroidism. Physical examination showed significant digital clubbing and laboratory tests showed: anemia (hemoglobin: 9.0 and hematocrit: 27), platelet count: 912,000, ESR: 130, PCR: 75, ferritin: 548, protein electrophoresis: increased alpha 2, negative autoantibodies and normal hands and knees X-ray. Bone scintigraphy showed osteogenic reactions of a diffuse and heterogeneous pattern (Figs. 1 and 2) that could correspond to metabolic alteration of HOA, and then pneumological, gastroenterological and hematological evaluation was requested to investigate secondary hypertrophic osteoarthropathy. Subsequently, presented a CT scan of the chest and abdomen without changes, pulmonary function test: no changes, esophagogastroduodenoscopy: postoperative status of esophagocolonoplasty, colonoscopy: no changes; bone marrow biopsy: bone marrow hypercellular for age and bone trabeculae within normal limits. After ruling out the neoplastic and infectious causes, the patient remained symptomatic and with high inflammatory tests, and then started the plan used for primary HOA with hydroxychloroquine $400 \mathrm{mg} / \mathrm{day}$, colchicine $0.5 \mathrm{mg}$ $12 / 12$ hours and prednisone $0.5 \mathrm{mg} / \mathrm{kg} /$ day. After 3 months, the patient returned reporting pain improvement and normal inflammatory tests. One year after the first evaluation, the patient maintains a stable condition with only digital clubbing and stable laboratory tests.

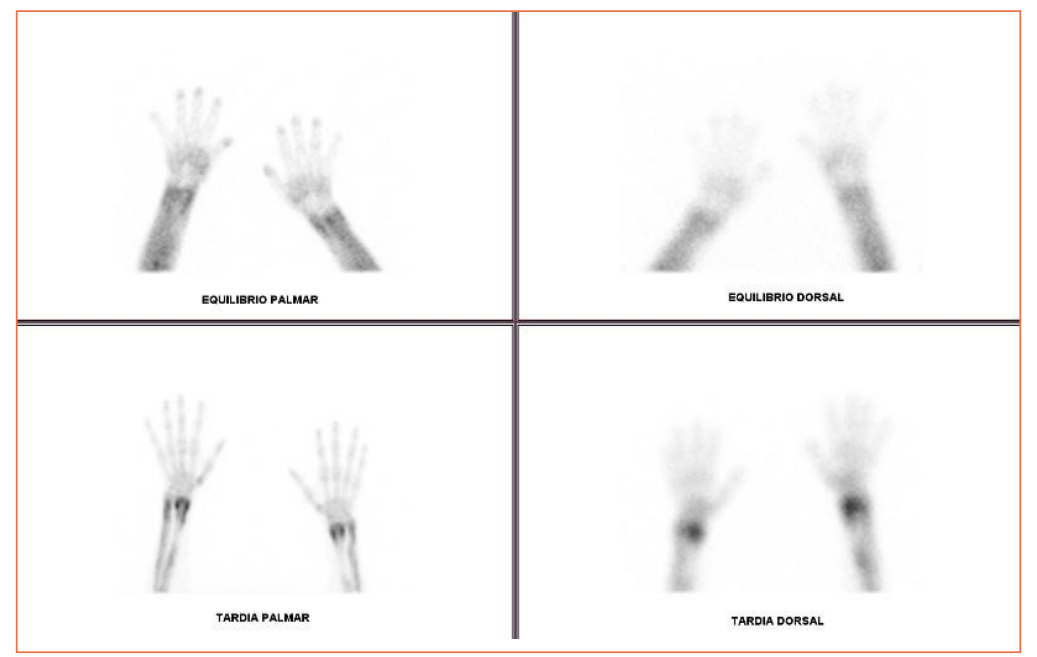

Figure 1. Bone scintigraphy with osteogenic reactions of a diffuse and heterogeneous pattern. 


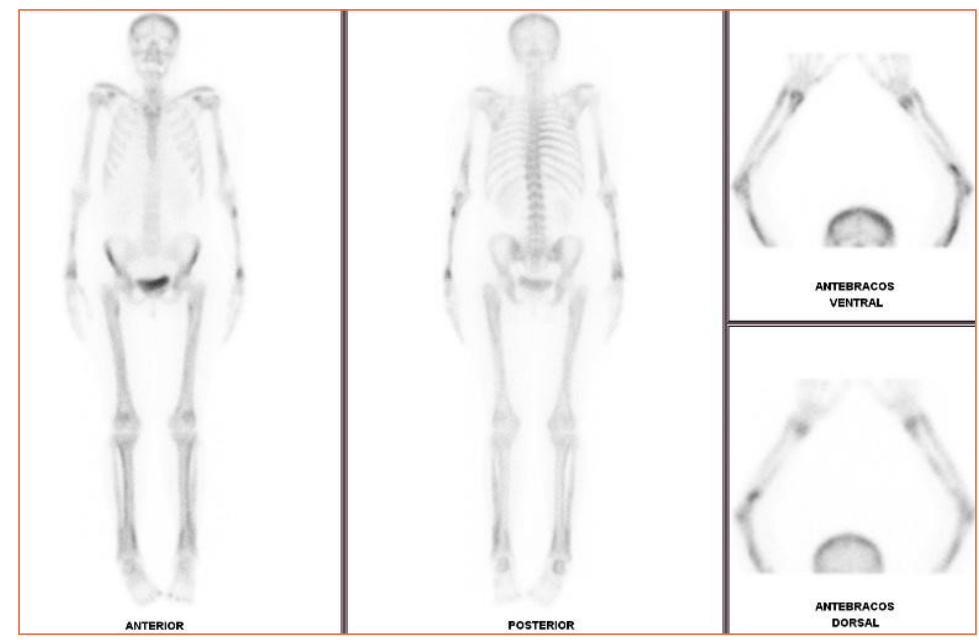

Figure 2. Bone scintigraphy with osteogenic reactions of a diffuse and heterogeneous pattern.

\section{CONCLUSION}

In a case suggestive of HOA, gastrointestinal, respiratory and hematological systems should be evaluated to identify the cause. In cases of idiopathic HOA, in which it is not possible to find a secondary and or genetic cause, there is the possibility of a response using medications for primary genetic HOA. 